\title{
Statistical power of the North Carolina Experiment III design in determining the likelihood of success of pedigree breeding programs in selfing plants
}

\author{
Eric M. Hallerman, \\ Jacques S. Beckmann $\dagger$ and \\ Morris Soller*
}

\author{
* Department of Genetics, The Hebrew University of \\ Jerusalem, 91904 Jerusalem, Israel. \\ $\dagger$ Department of Plant Genetics and Breeding, \\ Agricultural Research Organization, The Volcani \\ Center, 50250 Bet Dagan, Israel.
}

In plant pedigree breeding programs, predictions of the likelikood of program success with respect to improvement of a given quantitative character can be made on the basis of the phenotypic difference between parental lines, $2[d]$, and the additive genetic component, $D$, of the genetic variance of the quantitative character in the $F_{\infty}$ inbred products of the cross between them. Estimations of $D$ are subject to uncertainty, and as a consequence, breeding program recommendations may result in decision-making errors. The power of the North Carolina Experiment III design for estimating $D$ with regard to such errors was considered as a function of $[d]$ and true $D$, and of the size and family structure of the experiment. The optimal number of $F_{2}$ families and of replicates per family for the estimation of $D$ with high power was determined under various sets of genetic conditions. The sensitivity of the procedure to the underlying true value of $D$ and to the interval between critical decision-making thresholds was examined. Although the North Carolina Experiment III design proved robust under a wide range of genetic conditions, large experiments are required at borderline decision-situations when the difference in mean phenotypic expression between the parental lines crossed is small, while the likelihood of an equivocal decision is high when the difference between the parental lines crossed is large.

\section{INTRODUCTION}

Pedigree breeding programs for predominantly selfing plants are commonly based upon inbreeding of lines derived from $F_{2}$ individuals produced through the crossing of established pure lines. Methods have been developed to partition the variance of quantitative characters in $\mathrm{F}_{2}$ and backcross generations of a cross between two inbred lines into additive $(D)$, dominance $(H)$, and environmental $(E)$ components (Mather and Jinks, 1971), and to use the estimate of $D$ together with the observed difference between parental lines, $2[d]$, to make predictions regarding the likelihood of producing superior recombinant inbred lines by selfing the $F_{2}$ derivatives of such a cross (Jinks and Pooni, 1976). Kearsey (1980) has compared experimental designs appropriate for estimating the additive component of genetic variance, $D$, and has shown that the North Carolina Experiment III (Comstock and Robinson, 1952) is the most efficient.
The aim of the present study is to investigate the power of the North Carolina Experiment III (henceforth, NCIII) design in this situation; that is, to determine the likelihood that a recommendation regarding the continuation of a pedigree breeding program, based upon estimates of $D$ obtained through this design, will be correct. Power of the design will be considered as a function of the genetic architecture of the quantitative trait of interest in the parental lines, and of the size and family structure of the NCIII experiment devoted to estimation of additive genetic variance.

\section{THEORY}

\section{Estimation of the additive component of genetic variance}

Two different concepts of $D$, the additive component of genetic variance for a quantitative trait in the $F_{\infty}$ inbred products of a cross between two inbred lines, are at issue in assessing the reliability 
of predictions of success in a pedigree breeding program. These are true $D$ and estimated $D, \hat{D}$.

True $D$ is a precise parameter whose value is based upon the genetic architecture controlling expression of a quantitative trait, and is not known to the plant breeder.

The breeder's estimate of true $D, \hat{D}$, is an empirical value, which can be estimated on the basis of data from a set of crosses stemming from the two pure-breeding founder lines of the pedigree breeding program. In the NCIII design, each $\mathrm{F}_{2}$ individual is backcrossed to each of the original founder lines (Comstock and Robinson, 1952). Thus, the NCIII experiment consists of two backcross families for each of $k, \mathrm{~F}_{2}$ parents, each family comprising $r$ replicates. The additive component of genetic variance, $D$, is estimated (Kearsey, 1980) from the calculation of components of variance in an analysis of variance.

\section{Predicting the expected proportion of superior recombinant inbred lines}

The expected proportion of recombinant inbred lines that will be superior to the better-performing parental line (henceforth, proportion of superior recombinant inbred lines, PSRIL) depends not only upon the magnitude of true $D$, but also upon $2[d]$, the difference of mean expression of the quantitative trait between the parental lines (Mather and Jinks, 1971). For a new recombinant line to be considered superior to its betterperforming parent, the mean phenotypic expression of the given line would have to exceed the midparent value by at least $[d]$.

The expected distribution of the recombinant inbred lines derived from a cross between two parental lines with respect to a given quantitative trait will be normal, with mean equal to the midparent value and variance $D$ (Jinks and Pooni, 1976). The true PSRIL will be equal to the area of the standard normal distribution to the right of the value $z=[d] / D^{0.5}$. Thus, when $(d)$ and true $D$ are known, the decision whether or not to pursue a pedigree breeding program would depend upon the minimum expected PSRIL required for a positive decision. That is, one might decide to proceed only if the expected PSRIL were at least $0.05,0.01$, $0 \cdot 001$, or some other critical value.

\section{Confidence in program recommendations}

In practice, $[d]$ would be known, but true $D$ would not, and the decision to proceed ("go" decision) or not to proceed ("no go" decision) would be based upon the estimate of $D, \hat{D}$, as obtained from the NCIII (or other) experiment. Because of the difference between $\hat{D}$ and true $D$, however, the predicted PSRIL based upon the estimates obtained in any particular case will differ from the genetically determined "true" PSRIL. The recommended decision on the basis of $\hat{D}$ may therefore differ from that which would have been made had true $D$ been known. In particular, if $\hat{D}$ is greater than true $D$, the estimated PSRIL may be greater than the critical PSRIL value (i.e., critical with regard to decision making), in which case, one might proceed with the program when the true PSRIL is so small that a decision not to proceed would have been appropriate (Type I error). Alternatively, if $\hat{D}$ is less than true $D$, the estimated PSRIL may be less than the critical PSRIL value, so that one might decide not to proceed when a decision to proceed would have been appropriate (Type II error). Clearly, in any particular case, the likelihood of a Type I or Type II error will depend upon $[d]$, true $D$, the distribution and standard error of $\hat{D}$, and upon the critical PSRIL.

It is evident from the above that the closer the true PSRIL is to the critical PSRIL, the greater the likelihood of an error. Consequently, when the true PSRIL and the critical PSRIL are close to one another, avoiding decision errors will require that the standard error of $\hat{D}$ be very small, which in turn necessitates that the total experiment size, $N=2 k r$, be very large. Extension of this argument leads to the paradoxical conclusion that when the true PSRIL equals the critical PSRIL, an experiment of infinite size would be required in order to reach a correct decision! The resolution of the paradox appears to lie in the fact that at the critical PSRIL, the breeder has come to a conscious or unconscious estimate that the expected real costs and discounted benefits of the proposed breeding program are more or less equal. Consequently, in those genetic situations where the true PSRIL is at or near the critical PSRIL, the breeder is, in fact, indifferent as to whether or not to proceed with the breeding program. Thus, as the true PSRIL approaches the critical PSRIL, the costs (in terms of $N$ ) of making a correct decision tend to infinity, while the value of a correct decision tends to zero! Conversely, as the true PSRIL becomes greater or smaller than the critical PSRIL, the costs of making a correct decision decrease, while the value of a correct decision will increase.

These considerations lead to the definition of two critical PSRIL thresholds, upper and lower. The upper threshold is associated with a Type II error; that is, with coming to a "no go" decision 
when the true PSRIL is equal to or greater than the upper critical PSRIL $=b$. This would be considered an error, entailing loss of opportunity to obtain a superior inbred line. The lower critical PSRIL threshold is associated with a Type I error; that is, with coming to a "go" decision when the true PSRIL is equal to or less than the lower critical PSRIL $=a$. This would also be considered a serious error, entailing a large investment of breeding resources, with only a small likelihood of obtaining a superior recombinant inbred line. True PSRIL values that lie between the upper and lower critical PSRIL values represent a "grey area". For such values, either a "go" or "no go" decision are acceptable alternatives, and the breeder will have to make his decision on the basis of considerations other than the estimated PSRIL itself. If upper and lower critical PSRIL values are set too far apart, a large proportion of the estimates of $D$ may be in the grey area. In this case, the NCIII experiment would clearly not be fulfilling its function of providing decision-making guidance to the breeder.

It should be pointed out that the two possible types of decision-making error have asymmetrical consequences. A Type II error involves loss of resources already expended and the lost opportunity of producing a superior line. The consequences of a Type I error are greater-the unproductive commitment of limited resources, and the possible loss of reputation on the part of the breeder should the program ultimately fail. Thus, the risk that one is willing to accept of making a Type I error will be the primary factor determining experimental size.

\section{Estimation of experimental power}

In what follows, it will be assumed that the breeder will reach a "go" decision when $\hat{D}$ is such that the estimated PSRIL based upon $\hat{D}$ is equal to or greater than $b$, and will reach a "no go" decision when $\hat{D}$ is such that the estimated PSRIL based upon $\hat{D}$ is equal to or less than $a$. Given $[d]$, then, the value of $D, D_{b}$, which must be equalled or exceeded in order to recommend continuation of the breeding program according to the upper critical PSRIL, $b$, will equal:

$$
D_{b}=[d]^{2} / z_{b}^{2}
$$

where $z_{b}$ equals the standard score beyond which lies the area of the unit normal distribution equal to the upper critical PSRIL, $b$.

In an analogous manner, the value of $D, D_{a}$, at or below which discontinuation of the breeding program will be recommended according to the lower critical PSRIL, a, will equal:

$$
D_{a}=[d]^{2} / z_{a}^{2}
$$

where $z_{a}$ equals the standard score beyond which lies the area of the standard normal curve equal to the lower critical PSRIL, $a$.

If the estimate of $D, \hat{D}$, obtained in the NCIII design is greater than $D_{b}$, a "go" decision will be made. If $\hat{D}$ is less than $D_{a}$, a "no go" decision will be made. Comparison of the recommendation obtained on the basis of $\hat{D}$ to that which would have been made had true $D$ been known will enable the power of the Jinks and Pooni (1976) procedure against Type I or Type II error to be calculated as a function of $D, k$, and $r$.

Based on Jayaratnam and Graybill (1980), the power of an NCIII experiment against Type II error (i.e., the likelihood that $\hat{D}$ is less than $D_{a}$ when in reality true $D$ is equal to $D_{b}$ ) can be estimated by calculating

$$
\chi_{\mathrm{df}=k-1}^{2}=4 V_{1}(k-1) /\left(r D_{a}+4 V_{2}\right)
$$

where

$$
V_{1}=V_{2}+2 r D_{b} / 8
$$

is the expected mean square for additive effects in the NCIII ANOVA, and $k, r$, and $D_{a}$ are as previously defined, and $V_{2}$ is the expected mean square for error in the NCIII ANOVA, and will equal (Mather and Jinks, 1971, p. 140):

$$
V_{2}=D_{b} / 8+H_{b} / 8+V_{E}
$$

where $H_{b}=$ dominance component of variance, and $V_{E}=$ environmental component of variance. Given $k, r$, and true $D$ equal to or greater than $D_{b}$, the power of the experiment will equal the proportional area of the $\chi_{\mathrm{d} f=k-1}^{2}$ dsitribution from $-\infty$ to the calculated value of $\chi^{2}$.

Calculations of power for Type I error (i.e., the likelihood that $\hat{D}$ is equal to or greater than $D_{b}$, when in reality true $D$ is equal to $D_{a}$ ) are made as above except that

$$
\chi_{\mathrm{df}=(k-1)}^{2}=4 V_{1}(k-1) /\left(r D_{b}+4 V_{2}\right),
$$

and

$$
V_{1}=V_{2}+2 r D_{a} / 8
$$

and the power of the given NCIII experiment against Type I error will equal the proportional area of the $\chi_{\mathrm{df}=k-1}^{2}$ distribution from the calculated value of $\chi^{2}$ until $+\infty$. Areas of the $\chi^{2}$ distribution were obtained by interpolation of the tabulated values given by Pearson and Hartley (1972). 
Consideration of expressions (3), (4), (5), (6) and (7) shows that $V_{1}$ and $\chi_{2}$ will be primarily functions of $r, D_{a}$, and $D_{b}$, and are only affected marginally by the value of $V_{2}$. Thus, while $V_{2}$ will depend to some extent upon the degree of dominance, power calculations generally gave virtually identical results, whether codominance or complete dominance were assumed. For this reason, calculated results are presented only for the case of complete dominance $(H=D)$, which is a bit more conservative. Best results were generally checked by calculating for the case of codominance $(H=0)$ as well. In all instances, results (i.e., $\chi^{2}$ or power) were the same when rounded to two decimal places.

\section{Determination of optimal experimental design}

Optimal experimental design is generally defined as that combination of $k, F_{2}$ parents and $r$, replicates, which minimises $N$ for given experimental power. For any given true $D$ and upper and lower PSRIL (and hence, $D_{a}$ and $D_{b}$ values), different combinations of $k$ and $r$ yield different power estimates. The statistical power of particular combinations of $k$ and $r$ for given $D, D_{a}$, and $D_{b}$ can be determined by making appropriate substitutions into expressions (3), (4), (5), (6) and (7). A power curve for given $D, D_{a}$ and $D_{b}$ and $k$ is generated by carrying out this procedure using progressively larger values of $r$, starting with $r=2 ; r$ being allowed to increase until reasonable power is obtained, or until $N=2 k r$ reaches impractical size. To determine the optimal combination of $k$ and $r$ for given $D, D_{a}$ and $D_{b}$, such power curves are generated for values of $k$ ranging from 2 to some practical upper limit. The optimal $k, r$ combination, corresponding to the minimum value of the function, can be readily determined.

In practice, true $D$ is not known and can potentially take a wide range of values depending upon the parental lines and traits involved in the breeding program. Thus, optimal experimental design must be considered in terms of some particular assumed value of $D$ of special interest. In this study, we have chosen to consider experimental design for cases where true $D$ is equal to the marginal "go" or "no go" values, $D_{b}$ or $D_{a}$, and have calculated Type I power curves for true $D=$ $D_{a}$ and Type II power curves for true $D=D_{b}$. Since upper and lower PSRIL are a function of both the difference between parental lines, $2[d]$, and $D$, this means that the critical upper and lower PSRIL, $D_{a}$ and $D_{b}$, will be functions of 2[d]. This has the interesting consequence that critical upper and lower PSRIL, power curves for true $D$ equal to those critical limits, and optimal experimental design will depend upon the difference between parental lines, $2[d]$.

\section{Approximation of experimental power assuming normal distribution of $D$}

It may prove useful to note that for the values of $k$ and $r$ examined in this study, fairly close approximation of experimental power can be made by assuming that $\hat{D}$ is normally distributed about true $D$. The standard error of $\hat{D}, S E_{\hat{D}}$, is calculated (Kearsey, 1980) as:

$$
S E_{\hat{D}}=\sqrt{\frac{32}{r^{2}} \frac{V_{1}}{(k-1)} \frac{V_{2}}{2 k(r-1)}}
$$

where $V_{1}, V_{2}, k$, and $r$ are defined as above.

Following the above assumption, design of an NCIII experiment aimed at estimating $D$ with maximum precision for given experiment size $N=$ $2 k r$ entails adjusting $r$ and $k$ to minimise $S E_{\hat{D}}$. Given $[d]$ and true $D$, the probability of obtaining a value of $\hat{D}$ equal to or greater than $D_{b}$ will equal the proportion of the standard normal distribution to the right of $z_{b}$, where

$$
z_{b}=\left(D_{b}-D\right) / S E_{\hat{D}}
$$

and the probability of obtaining a value of $\hat{D}$ equal to or less than $D_{a}$ will equal the proportion of the standard normal distribution to the left of $z_{a}$, where

$$
z_{a}=\left(D_{a}-D\right) / S E_{\hat{D}} .
$$

\section{NUMERICAL RESULTS}

\section{$P S R I L$ values in relation to the difference between parental lines and additive genetic variance}

A contour graph (fig. 1) was constructed, showing curves of equal given PSRIL values, as a function of half the difference between parental lines, $[d]$, and the additive genetic variance, $D$. Contours of equal PSRIL were obtained by substituting values of $[d]$ into the expression:

$$
D=(d)^{2} / z_{\text {PSRIL }}^{2}
$$

where $z_{\text {PSRIL }}$ equals the standard score corresponding to the point on the abscissa to the right of which the area of the standard normal curve equals the given PSRIL. For example, when $[d]=2 \cdot 0$, obtaining a PSRIL $=0.01$ (hence, $z=2.33$ ), requires true $D=2^{2} /(2 \cdot 33)^{2}=0 \cdot 736$. Both $[d]$ and 


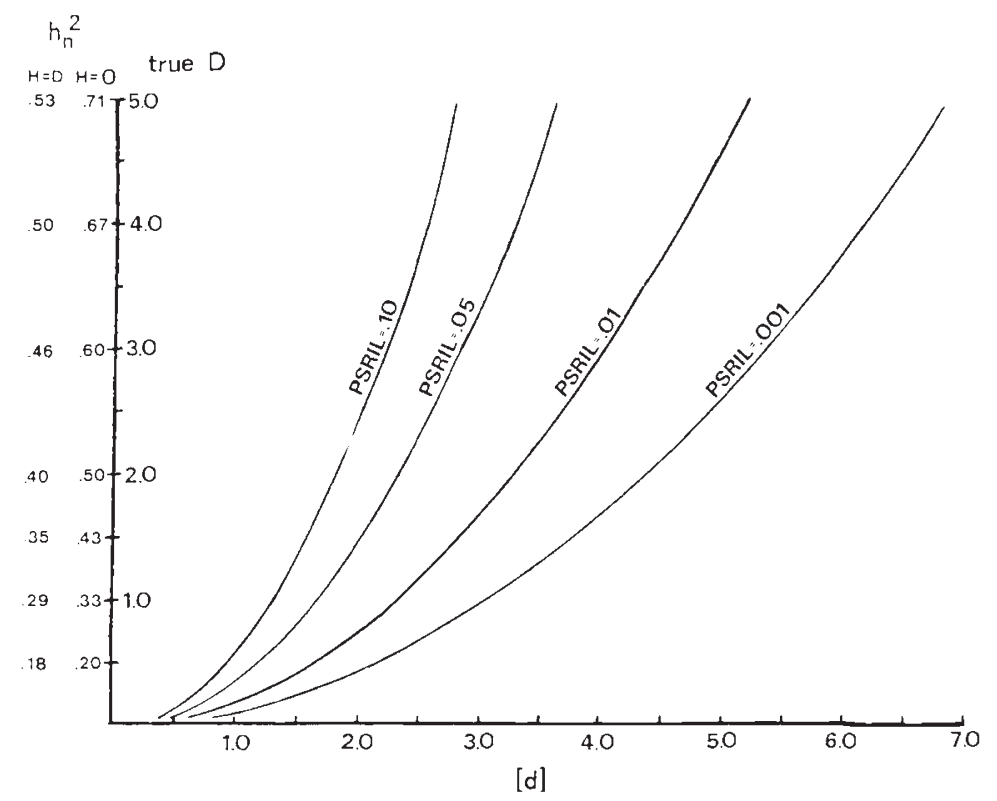

Figure 1 True PSRIL as a function of the difference between parental lines $[d]$ and the additive genetic variance, $D$, in units of the within-line phenotypic standard deviation. The relationships of $h_{n}^{2}, D$ and $H$ are as described in the text.

$D$ are given in units of $\sigma_{E}$, i.e., the standard deviation within the parental lines or the $F_{1}$. In this case, the (narrow) heritability, $h_{n}^{2}$, of the trait in the $\mathrm{F}_{2}$ is simply $h_{n}^{2}=D /(D+H / 2+2)$. This is shown as a second scale along the $D$ axis in fig. 1, separately for $H=0$ (no dominance) and $H=D$ (complete dominance). As noted above, although $D$ is biologically independent of $[d]$, for a given PSRIL value, $D$ is a strict function of $[d]$.

As the difference between parental lines increases, the additive genetic variance required for given PSRIL increases exponentially. For example, when $[d]=1 \cdot 0, D=0 \cdot 18$ will yield PSRIL $=0.01$, while $D=2.94$ is required when $[d]=4 \cdot 0$. Thus, these curves express the truism that the more the lines differ, the greater must be the additive genetic variance in order to obtain a given proportion of superior recombinant inbred lines. A corollary of this relationship, however, has an important decision-making consequence. Namely, the more the lines differ, the greater the difference between the $D$ values corresponding to a given of upper and lower critical PSRIL. For example, at $[d]=4 \cdot 0, D_{0.001}=1.67$ and $D_{0.01}=$ $2 \cdot 94$, while at $[d]=1 \cdot 0, D_{0.001}=0.10$ and $D_{0.01}=$ $0 \cdot 18$. Thus, when the difference between parental lines is large, a large additive genetic variance is required to yield an attractive PSRIL value, but it will be easy to distinguish experimentally between marginal "go" and "no go" situations. Conversely, however, when the difference between parental lines is small, it does not require a great deal of additive genetic variance to yield an attractive PSRIL, but it will be difficult to distinguish experimentally between a true "no go" situation, and a ture, but marginal, "go" situation. Exactly opposite conclusions hold with respect to the "grey" area. Consideration of fig. 1 shows that when $[d]$ is small, the grey area is also small. Thus, in this case, the NCIII experiment, if sufficiently large, should almost always allow a decision to be made. Conversely, when $[d]$ is large, the grey area is also large. Thus, in this case, the NCIII experiment may not infrequently yield estimates of $D$ that fall within the grey area and do not allow an experimentally-based decision.

\section{Power in relation to the difference between parental lines}

The above considerations are illustrated in fig. 2, which shows power curves against Type I and Type II errors for the cases $[d]=1.0$ and $[d]=2 \cdot 0$, as a function of increasing replicate size $r$ (and hence, increasing experiment size, $N=2 k r$ ) given an NCIII experiment based upon $k=50 \mathrm{~F}_{2}$ parents and assuming that true $D$ equals $D_{a}$ or $D_{b}$ for Type I and Type II errors, respectively. The cases considered here are the marginal cases, and hence will require larger experiments for given power than cases where $D$ is less than $D_{a}$ or $D$ is greater than $D_{b}$. Since $D_{a}$ and $D_{b}$ are functions of $[d]$, power curves for $[d]=1 \cdot 0$ and $[d]=2 \cdot 0$ are expec- 


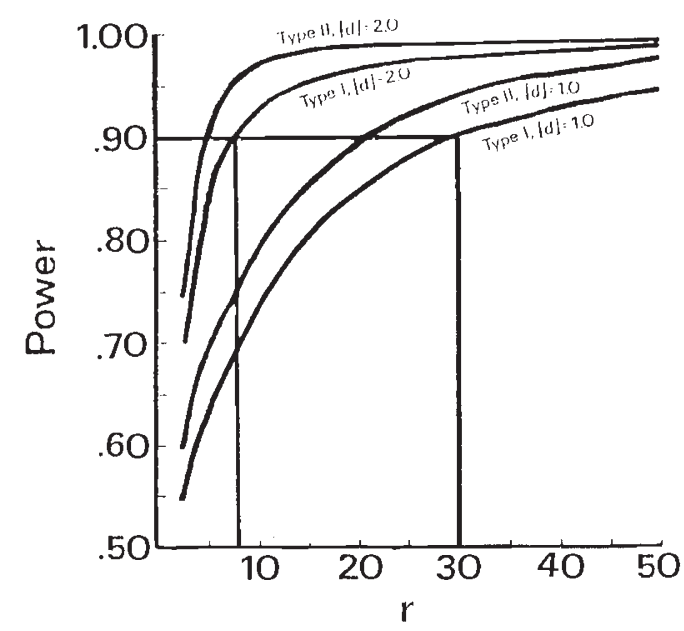

Figure 2 Power against Type I and Type II decision making errors as a function of number of replicates, $r$, in a $k=50$ parent NCIII experiment for $[d]=2.0$ and $[d]=1 \cdot 0$.

ted to differ.

The calculation of power against Type I error for a particular $r(=20)$, with $[d]=1.0$ and $k=50$ as set out above, illustrates construction of the figure. Since this is a Type I error situation, we ask the question "If true $D$ is such that it gives rise to a true PSRIL equal to $0 \cdot 001$, what is the likelihood that this experiment will lead to a $\hat{D}$ and corresponding estimated PSRIL equal to or greater than 0.01 , i.e., large enough to recommend continuation of the breeding program?" Substituting into (2) and (1) with $z_{\text {PSRIL }=0.001}=3.09$ and $Z_{\mathrm{PSRIL}=0.01}=2 \cdot 33$, gives $D_{a}=0.1047$ and $D_{b}=$ $0 \cdot 1842$, respectively. Substituting into (4) and (5) with $D=D_{a}$ and $V_{E}=1 \cdot 0$, gives $V_{2}=1.0262$ (complete dominance), and $V_{1}=1.5497$. Substituting into (6) gives $\chi_{\mathrm{dr}=49}^{2}=38.99$. Looking under 49 degrees of freedom in the table of $\chi^{2}$ and interpolating from adjacent values, the proportional area of the distribution from this value of $\chi^{2}$ until $+\infty$ yields 0.842 , which will be the Type I power of this particular experiment at the stated conditions.

In general, power curves for the case $[d]=2 \cdot 0$ are steeper and higher than those for $[d]=1 \cdot 0$. That is, for $[d]=2 \cdot 0$, power against both error types increases more rapidly with experiment size and is at consistently higher levels than for $[d]=$ 1.0. Consider, for example, a Type I error situation; i.e., true $D$ is equivalent to a PSRIL $=0.001$, but a "go" decision will be made if $\hat{D}$ is greater than $D_{b=0.01}$. For $[d]=2 \cdot 0$, a power of 0.90 against this sort of error will be reached at $r=8(N=800)$; for $[d]=1 \cdot 0$, at $r=30(N=3000)$, while a power of 0.95 in this situation can be obtained at $r=15$ $(N=1500)$ for $[d]=2 \cdot 0$, and at $r=54(N=5400)$ for $[d]=1 \cdot 0$.

Similarly, when considering a type II error situation, true $D$ is equivalent to a PSRIL $=0.01$, but a "no go" decision will be made if $\hat{D}$ is less than $D_{a=0.001}$. Power of 0.90 will be obtained for $[d]=2 \cdot 0$ at $r=5(N=500)$, but for $[d]=1.0$ only at $r=21(N=2100)$; while power of 0.95 will be obtained at $N=800$ and $N=3300$, respectively.

Fig. 2 also shows that power curves for Type II errors are higher and steeper than for Type I errors. This is a consequence of the fact that $D_{b}$ and hence $S E\left(D_{b}\right)$ are always greater than $D_{a}$ and $S E\left(D_{a}\right)$. Other things being equal, the breeder would generally set $k$ and $r$ so as to obtain the desired power against the more serious Type I error. Some additional degree of experimental power would consequently be obtained for Type II error.

\section{Experimental power in relation to the true additive genetic variance, $D$}

As pointed out above, critical $D_{a}$ and $D_{b}$ values are closer together when the difference, $2[d]$, between parental lines is small, and hence, in such a case, a larger experiment is required to distinguish the marginal alternatives with good power. Power as calculated above was for situations where true $D$ was such that true PSRIL were equal to their corresponding critical PSRIL. These are the cases where the analysis is at its weakest. In reality, as pointed out previously, true $D$ is not dependent upon $[d]$ and can vary freely: power against Type I error increasing the more true $D$ is greater than $D_{b}$, and power against Type II error increasing the more true $D$ is less than $D_{a}$. These effects are shown in fig. 3 , which shows power curves for Type I and Type II errors for $[d]=1.0$ and $[d]=2.0$ as true $D$ exceeds $D_{b}$ or becomes lower than $D_{a}$. For these cases, experimental size for each $[d]$ was taken as the optimal combination of $k$ and $r$ as given in table 1 for power $=0.90$ against Type I error when true $D=D_{b=0.01}$.

As an illustrative example, consider calculation of power against Type II error for the case $[d]=2 \cdot 0$ and true $D=0.85$, with upper PSRIL, $b=0.01$. From table 1 , optimum $k$ and $r$ for $[d]=2.0$ are 73 and 5, respectively. Substituting into (1) and (2) with $z_{\mathrm{PSRIL}=0.01}=2.33$ and $z_{\mathrm{PSRIL}=0.001}=3.09$ gives $D_{b}=0.7368$ and $D_{a}=0.4189$, respectively. Substituting into (4) with $D=0.85, V_{2}=1 \cdot 0$, and $r=5$ gives $V_{1}=2 \cdot 2750$. Substituting into (3) with $k=73$ gives $\chi^{2}=94 \cdot 35$. Looking under $(k-1)=72$ degrees of freedom in the $\chi^{2}$ table and interpolating from adjacent values gives power against Type II error of 0.96 . 


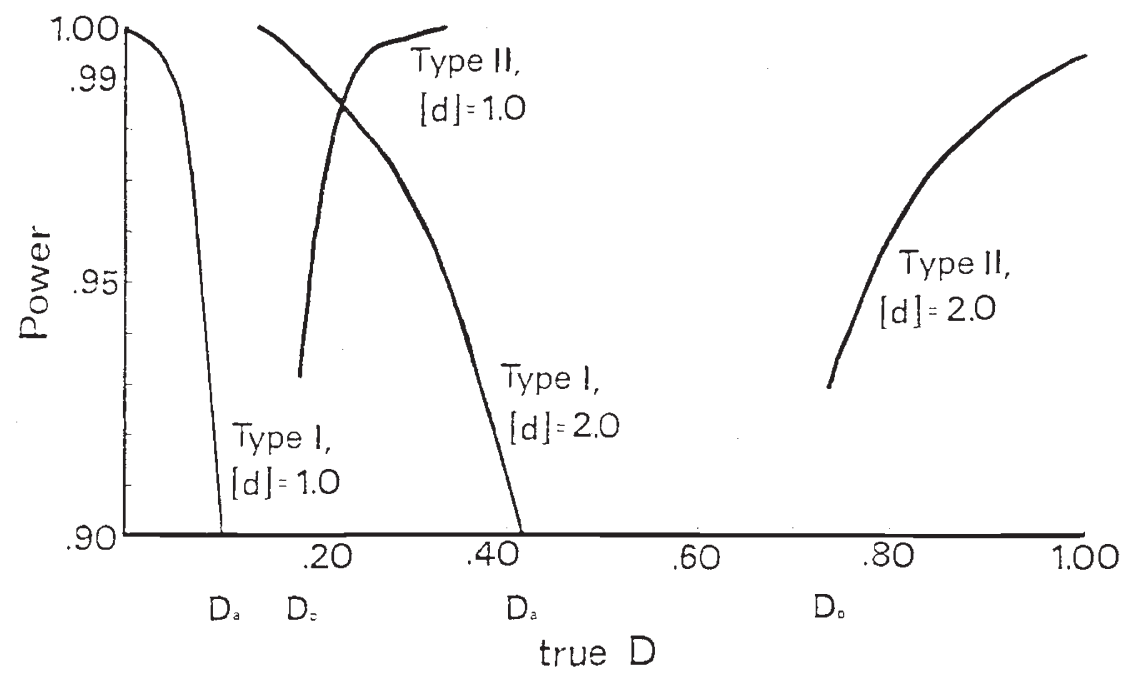

Figure 3 Power of the NCIII experiment as a function of the true additive genetic variance, $D$.

In fig. 3 , it can be seen that in all curves, power increases very steeply as true $D$ values deviate in the indicated directions from the critical values defining the grey area. Power changes more steeply at $[d]=1 \cdot 0$ than at $[d]=2 \cdot 0$, but the difference is not great. Thus, these calculations show that an experiment planned to give good power for $D$ equal to the marginal $D_{a}$ and $D_{b}$ values will give very high power when true $D$ values are greater than $D_{b}$ or less than $D_{a}$.

When true $D$ lies between $D_{a}$ and $D_{b}$, the likelihood of obtaining an estimate of $D$, $\hat{D}$, in the grey area is maximal. Alternatively, values of $\hat{D}$ greater than $D_{b}$ or less than $D_{a}$ may also be obtained, both with considerable likelihood. In the latter instance, a "decision" will be provided by the procedure described here, but it will be the equivalent to a decision based upon tossing a coin.
Clearly, the larger the grey area, the more likely it is that true $D$ will fall within its limits, with corresponding weakness of the prediction procedure.

\section{Experimental size for given power, critical $P S R I L$, and parental line difference, in relation to the number of parents and number of replicates per backcross family in the NCIII design}

As pointed out in the Theory section, power for any given $[d], D$ and critical PSRIL is determined by the critical $D_{a}$ and $D_{b}$ values implied by [d] and the PSRIL and by the distribution of $\hat{D}$, which in turn, is a complex function of number of parents, $k$, tested in the NCIII design and number of replicates per family, $r$. Consequently, for any given [d], $D$ and set of critical PSRIL, there will be an

Table 1 Number of $F_{2}$ parents $(k)$, and number of replicates per backcross family $(r)$, yielding smallest experiment size $(N)$, for NCIII experiments yielding given levels of experimental power as a function of difference between parental lines, $[d]^{*}$

\begin{tabular}{|c|c|c|c|c|c|c|c|c|c|c|c|c|}
\hline \multirow{2}{*}{$\begin{array}{l}\text { Difference } \\
\text { between parental } \\
\text { lines, }[d]\end{array}$} & \multicolumn{3}{|c|}{$\begin{array}{l}\text { Type I error } \\
\text { Power }=0.90\end{array}$} & \multicolumn{3}{|c|}{ Power $=0.95^{\dagger}$} & \multicolumn{3}{|c|}{$\begin{array}{l}\text { Type II error } \\
\text { Power }=0 \cdot 90\end{array}$} & \multicolumn{3}{|c|}{ Power $=0.95$} \\
\hline & $k$ & $r$ & $N$ & $k$ & $r$ & $N$ & $k$ & $r$ & $N$ & $k$ & $r$ & $N$ \\
\hline $1 \cdot 0$ & 69 & 21 & 2898 & $\begin{array}{r}92 \\
100\end{array}$ & $\begin{array}{l}25 \\
23\end{array}$ & $\begin{array}{l}4600 \ddagger \\
4600 \ddagger\end{array}$ & 27 & 36 & 1944 & 54 & 31 & 3348 \\
\hline 1.5 & 72 & 9 & 1296 & 102 & 10 & 2040 & 27 & 16 & 864 & 53 & 14 & 1484 \\
\hline $2 \cdot 0$ & 73 & 5 & 730 & 115 & 5 & 1150 & 27 & 9 & 486 & 60 & 7 & 840 \\
\hline 3.0 & 82 & 2 & 328 & $\begin{array}{r}86 \\
129\end{array}$ & $\begin{array}{l}3 \\
2\end{array}$ & $\begin{array}{l}516 \ddagger \\
516 \ddagger\end{array}$ & 27 & 4 & 216 & 63 & 3 & 378 \\
\hline $4 \cdot 0$ & 47 & 2 & 188 & 65 & 2 & 260 & 31 & 2 & 124 & 53 & 2 & 212 \\
\hline
\end{tabular}

* In these calculations, it is assumed that the additive $\mathrm{F}_{2}$ genetic variance (true $D$ ) is equal to $D_{b}$ for Type I error calculations, and to $D_{a}$ for Type II error calculations. See text for details.

$\dagger \chi^{2}$ values for degrees of freedom (df) greater than 100 were estimated as: $\chi^{2}=\mathrm{df}-1.56(2 \mathrm{df})^{0.5}$, after Walpole and Myers (1978). $\ddagger$ For $[d]=1.0$ and $[d]=3 \cdot 0$, alternative experiments of equal size yield power $=0.95$ against Type I error. 
optimal $k$ and $r$ that will provide desired power at minimum total experiment size $N=2 \mathrm{kr}$.

In order to explore this question, Figure 4 was prepared for $[d]=2 \cdot 0$ and $[d]=1 \cdot 0$, showing total experiment size, $N$, required to provide power of 0.90 against both type I and Type II errors as a function of $k$, given upper and lower critical PSRILs $b=0.01$ and $a=0.001$, respectively. Type I error was calculated for true $D$ equal to $D_{a}$, and Type II error was calculated for true $D$ equal to $D_{b}$. For each set of genetic conditions, power curves were calculated for all values of $k$ from 2 to 100 . For each particular value of $k$, power was calculated for $r$ between 2 and 150. In each case, the optimum $k$ value giving minimum $N$ is indicated. Although optimum $k$ values differed greatly between error-types, and also between $[d]=2 \cdot 0$ and $[d]=1 \cdot 0$, it is clear that $k$ can vary quite widely in both directions from the optimum for Type I error, and in the up direction for Type II error, without having appreciable effects upon total experiment size. Furthermore, it also appears that $k$ values in the range $40-80$ will be close to optimal in most situations. The "sawtoothing" effect in each of the curves was the consequence of consecutive values of $k$ requiring the same number of replicates in order to achieve power $=0.90$, giving increasing $N=2 k r$ until at a particular $k$, the number of required replicates decreased by one, with $N=2 k r$ then decreasing.
Table 1 shows optimal values of $k, r$, and $N$ for a range of values of $[d]$, given upper and lower critical PSRIL, $b=0 \cdot 01$ and $a=0 \cdot 001$, respectively, power of either 0.90 or 0.95 for both Type I and Type II errors, and assuming true $D$ equals $D_{a}$ and $D_{b}$ for Type I and Type II errors, respectively. Note again that although there is no correlation between $(d)$ and true $D$, the critical values $D_{a}$ and $D_{b}$ are functions of [ $\left.d\right]$, so that values of $k, r$, and $N$ in table 1 change with the magnitude of [d]. As noted previously, required values of $N$ for given power increase markedly at small values of $[d]$. This is primarily a consequence of an increase in the optimal number of replicates per family.

Total experimental size under optimum $k$ and $r$ required for power of 0.95 as compared to a power of 0.90 stand in more or less constant proportion of 1.57 . This is approximated by the ratio of their respective $z$ values. Similarly, total experiment size for Type I as compared to Type II error stand in more or less constant proportion of 1.72 , again approximated by the relation of the respective $z$ values for $D_{a}$ as compared to $D_{b}$.

\section{The effect of narrowing the decision-making thresholds}

When the interval between critical PSRILs was narrowed by half (from $a=0.001$ and $b=0.01$ to $a=0.003$ and $b=0.008$ ), minimum experiment

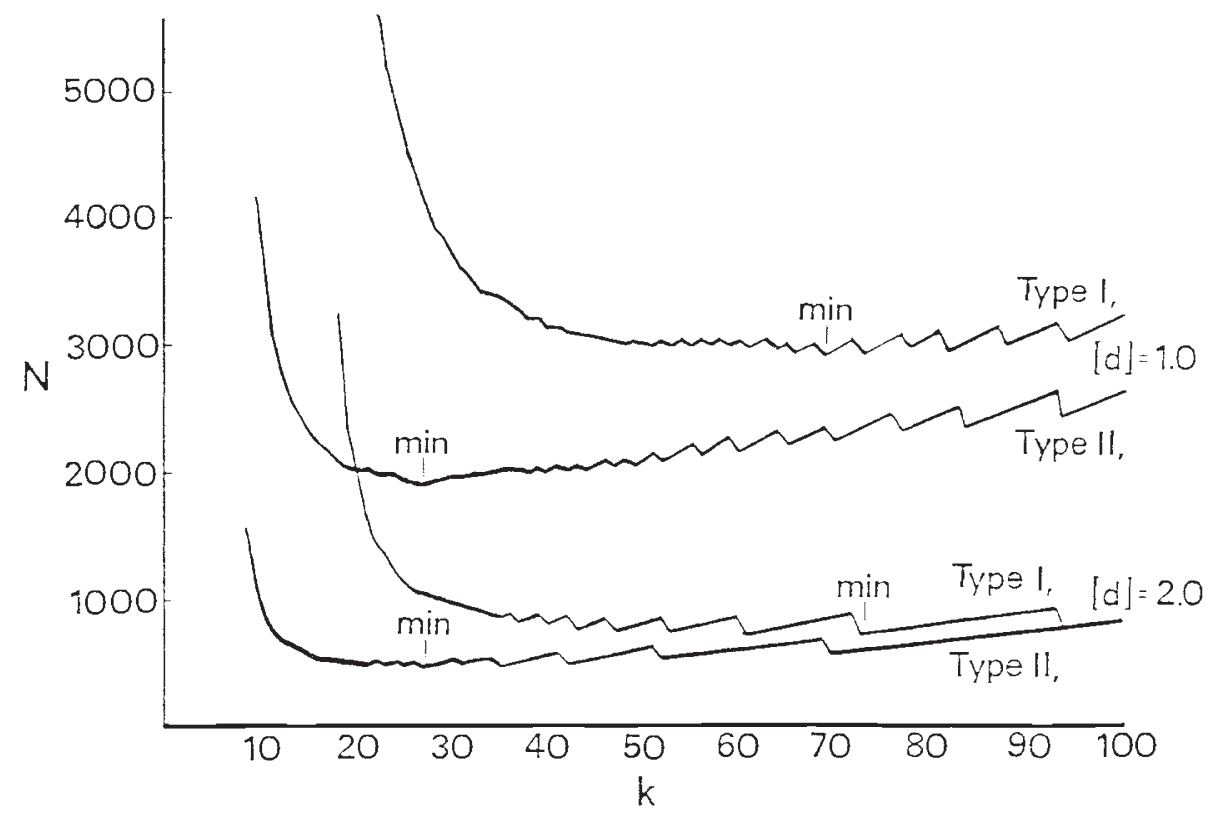

Figure 4 Minimum NCIII experiment size yielding power of 0.90 against both Type I and Type II error as a function of the number of $\mathrm{F}_{2}$ parents, $k$, for $[d]=2 \cdot 0$ and $[d]=1 \cdot 0$. 
size for power $=0.90$ increased almost threefold (data not shown). Thus, as the decision making thresholds are brought closer together, much larger experiments are needed to maintain a desired level of confidence. Consequently, narrowing the critical thresholds beyond the point taken in this study would seem to be inadvisable from the standpoint of experimental design, except when $[d]$ is large. In this case, optimum experimental size for the given PSRIL is small, and narrower thresholds may be desired in order to narrow the "grey" area.

\section{Estimation of experimental power following the assumption that $\hat{D}$ is normally distributed about true $D$}

Calculations based upon the assumption of a normal distribution of $\hat{D}$ about true $D$ lead to an underestimation of experimental power against Type I error, and an overestimate of power against Type II error. Optimal experiments aimed at providing power $=0.90$ (or 0.95 ) against Type I error were approximately 12 per cent (or 19 per cent) larger than those required by the more exact method. Those aimed at providing power $=0.90$ (or 0.95) against Type II error were approximately 5 per cent (or 10 per cent) smaller than those required by the more exact method.

\section{DISCUSSION}

Jinks and Pooni (1976) suggested the use of the additive genetic variance, $D$, of the inbred products of a cross between two inbred parental lines as a means of determining the potential of a pedigree breeding program based upon such a cross. Kearsey (1980) further compared experimental designs for estimation of $D$, and developed a method of minimising standard errors for given experiment size. The fact that estimates of $D$ do have a standard error, however, necessarily implies that decisions based upon these estimates have a possibility of error. In the present study, two possible errors were defined: Type I error, in which the true situation is one in which the breeding program would have a low probability of generating a superior recombinant inbred line (e.g., PSRIL $=0.001$, yet on the basis of the experimental estimate of $D$, a decision is made to proceed with the program; and a Type II error, in which the true situation is one in which the breeding program would have a reasonable probability of success (e.g., PSRIL $=0.01$ ), yet on the basis of the experimental estimate of $D$, a decision is made to discontinue the program. These error types are analogous to the Type I and Type II error of statistical tests of hypotheses.
The power of the North Carolina Experiment III (NCIII) design against each of these error types, i.e., the likelihood that a decision based upon the NCIII-derived estimate of $D$ would be correct, was explored in relation to the phenotypic difference between parental lines, the true $D$ of their inbred products, and the magnitude and family structure of the NCIII experiment devoted to the estimate of $D$. The salient conclusions of this study can be summarized as follows:

\section{Upper and lower critical decision making thresholds}

In order to make effective use of $D$ estimates in deciding whether to continue a particular pedigree breeding program, the breeder must accept that he will not be able to define an experiment that can yield a clear-cut, error-free decision; and that to define his error situation, it is necessary to define both upper and lower critical PSRIL thresholds: i.e., the proportion of superior recombinant inbred lines that would make the pedigree breeding program rewarding, and the corresponding proportion that would make the program unrewarding. Given upper and lower critical PSRIL, it will be possible to design experiments that provide desired power when the true genetic situation is at one or the other or these limits, and better than desired power when the true genetic situation is beyond these limits. The area between these limits is defined as a "grey" area, in which the breeder is essentially indifferent as to whether he makes a "go" or "no go" decision. That is, the breeding program is sufficiently large so as to give a good chance of retrieving a superior inbred, even if the expected PSRIL is less than 0.01 (although greater than 0.001 ), but it would be preferable to turn the available resources to programs where the expected number of superior inbreds is greater. If the value of true $D$ lies within the grey area, this means that within the limits of the experiment, there are good chances of getting either a "go" or a "no go" decision, and that in spite of the experiment devoted to estimating $D$, the breeder is essentially tossing a coin in deciding which way to proceed.

The upper and lower critical PSRIL thresholds adopted in this study were $b=0.01$ and $a=0.001$, respectively. That is, it is assumed that the breeder would not want to discontinue a program if the expected PSRIL were 0.01 or higher; he would not want to continue the program if the expected PSRIL were 0.001 or lower; and he would be indifferent as to whether to continue or not if the expected PSRIL were between 0.01 and 0.001 . Poisson considerations show that the expected 
number of superior recombinant inbred lines should be on the order of 4-5 in order to have a high probability that at least one or two such lines will actually be found. Thus, a program with PSRIL of 0.001 would require raising and screening about 5000 recombinant inbred lines out of the initial cross. Although many lines would be discarded in the initial stages of the program, the program would still have to be of considerable magnitude to allow for the production of many hundreds of lines for the final testing stages. Thus, a PSRIL of 0.001 seems to be as low as one could go for the lower critical threshold. Similarly, a program that would yield an expected number of 10 superior inbreds in a program of 1000 initial lines would seem to be one that had sufficient promise of success to be continued. Thus, an upper critical PSRIL of 0.01 seems a reasonable choice. As shown in the Numerical Results Section, the range between upper and lower critical PSRIL cannot be narrowed appreciably without necessitating a drastic increase in experimental size.

\section{Family structure of an NCIII experiment}

Although optimal family structure for an NCIII experiment would generally be that yielding minimum experiment size, practical constraints limiting $k$ might dictate the need to regard some alternative structure as operationally optimal. In strongly selfing plants, for example, it may be difficult to produce numerous backcross families, but each such family could readily include large numbers of individuals. In such cases, an experiment with smaller $k$ and somewhat larger $r$ than in the optimal case might be warranted.

\section{The effect of the phenotypic difference between parental lines on experimental size}

Experimental size required for given power was calculated on the assumption that true $D$ was equal to the critical marginal $D$ 's $\left(D_{a}\right.$ or $\left.D_{b}\right)$ required for given parental difference $[d]$ and upper and lower PSRIL. In reality, of course, true $D$ is a function neither of $[d]$ nor of the PSRIL (although it will tend to be larger as $[d]$ increases), and can be expected to vary over a wide range of values, depending upon the trait in question and the particular pair of parental lines compared. Because of the steep increase in power as true $D$ deviates from the critical values defining the grey areas, the Jinks and Pooni (1976) approach will often give a correct "go" or "no go" decision, even if experimental sizes are small, when true $D$ is greater than the upper critical value, $D_{b}$, or lower than the lower critical value, $D_{a}$. When $[d]$ is small, however, problems may arise for small experimental sizes when the NCIII experiment provides an estimate of $D$ indicating that there is enough additive genetic variance to make the program seem attractive, yet the possibility that this is due to sampling error from zero variance is high.

Alternatively, when $[d]$ is large, problems may arise when estimates of $D$ fall into the large grey area, in which case a firm recommendation cannot be made. In addition, when the grey area is large, there is a greater likelihood that true $D$ itself will fall within the grey area, in which case the predictive value of the Jinks and Pooni (1976) approach is weak. Thus, in planning a Jinks and Pooni (1976) experiment, rather large experimental sizes are required, if one wishes to reduce the possibility of falling into either of the above problem situations. In this context, it will be of interest to examine the power of the marker-based methods proposed by Soller and Beckmann (1983) and Beckmann and Soller (1986) for predicting the results of a pedigree-breeding program.

Acknowledgements The authors gratefully acknowledge the assistance of Esther Katz in development of the computer programs used in this study, and of Dr A. Genizi in development of the power calculations. Useful comments were contributed by the referee. This research was supported by a grant from the U.S.-Israel Binational Agricultural Research and Development Fund (BARD).

\section{REFERENCES}

BECKMANN, J. S., AND SOlLER, M. 1986. RFLPs in plant genetic improvement. In Miflin, B. J. (ed.) Oxford Survey of Plant Molecular and Cell Biology 3, Oxford Press, Oxford, U.K., pp. 196-250.

COMSTOCK, R. E., AND ROBINSON, H. F. 1952. Estimation of average dominance of genes. In Heterosis, Iowa State College Press, Iowa City, Iowa, chapter 30.

JAYARATNAM, S., AND GRAYBILL, F. A. 1980. Confidence intervals on variance components in three factor crossclassification models. Technometrics, 22, 375-380.

JINKS, J. L., AND POONI, H. S. 1976. Predicting the properties of recombinant inbred lines derived by single seed descent. Heredity, 36, 253-266.

KEARSEY, M. J. 1980. The efficiency of the North Carolina Experiment III and the selfing, backcrossing series for estimating additive and dominance variation. Heredity, 45 , $73-82$.

MATHER, K., AND JINKS, J. L. 1971. Biometrical Genetics. Chapman and Hall, London.

PEARSON, E. S., AND hARTLEY, H. D. 1972. Biometrika Tables for Statisticians, Vol. II. University Printing House, Cambridge, U.K.

SOLLER, M., AND BECKMANN, J. S. 1983. Genetic polymorphisms in varietal identification and genetic improvement. Theoretical and Applied Genetics, 67, 25-33.

WAlPole, R. E., AND MYERS, R. H. 1978. Probability and Statistics for Engineers and Scientists, 2nd ed. Macmillan Publishing Co., Inc., New York. 